\title{
ESTUDO EPIDEMIOLÓGICO DA MORTALIDADE PÓS NEONATAL ASSOCIADA A CAUSAS EVITÁVEIS EM PALMAS, TOCANTINS E BRASIL
}

Epidemiological study of post neonatal mortality associated with avoidable causes in Palmas, Tocantins and Brazil. 2014-2016

Estudio epidemiológico de la mortalidad poste neonatal asociada a causas evitables en Palmas, Tocantins y Brasil. $2014-2016$

Maria Luiza Silva Brito ${ }^{* 1}$, Daniel Botelho Mariano ${ }^{1}$, Ana Flávia de Oliveira Castro ${ }^{1}$, Renata dos Santos Oliveira ${ }^{1}$, Tatiane Pires Oliveira ${ }^{1}$, Antônio Rozeni Gomes Barbosa Júnior $^{1}$, Karolyne Botelho Marques Silva ${ }^{2}$, Glêndara Aparecida de Souza Martins ${ }^{3}$, Sandra Maria Botelho Mariano ${ }^{4}$

${ }^{1}$ Acadêmico (a) do curso de Medicina, Universidade Federal do Tocantins (UFT), Palmas, Brasil.

${ }^{2}$ Coordenadora do Programa de Saúde da Família e Comunidade da Secretaria Municipal de Saúde - Palmas Tocantins, Brasil.

${ }^{3}$ Professora Doutora do Curso de Engenharia de Alimentos da Universidade Federal do Tocantins (UFT), Palmas, Brasil.

${ }^{4}$ Professora Doutora do Curso de Medicina da Universidade Federal do Tocantins (UFT), Palmas, Brasil.

*Correspondência: Laboratório experimental, Bloco I, sala 02, Universidade Federal do Tocantins, Avenida NS15, Quadra 109, Norte, s/n - Plano Diretor Norte, Palmas - TO, 77001-090. e-mail:Sandrabotelho@uft.edu.br

Artigo recebido em 21/11/-2018 aprovado em 18/06/2019 publicado em 02/10/2019.

\section{RESUMO}

A mortalidade infantil, em especial a pós neonatal, é um sensível indicador da qualidade de vida de uma população, sendo assim, objetivou-se apresentar o coeficiente de mortalidade pós neonatal da cidade de Palmas, do Estado do Tocantins e do Brasil, no triênio 2014-2016, além de expor as principais causas evitáveis destes óbitos. Trata-se de um estudo descritivo exploratório que analisa dados públicos disponibilizados pelo Sistema de Informações sobre Mortalidade (SIM) e Sistema de Informação sobre Nascidos Vivos (SINASC). Os resultados apresentaram como principais fatores vinculados à mortalidade pós neonatal a deficiência no diagnóstico e tratamento, em ações de promoção vinculadas a atenção primária, na atenção ao recém-nascido (RN), a gestação e ao parto, e imunizações. Encontrou-se uma redução significativa da mortalidade em Palmas-TO, o oposto dos panoramas Estadual e Nacional. As taxas de mortalidade apresentaram variações. Palmas e Tocantins aumentaram seu valor de 2014 (1,55/1.000 nascidos vivos (NV) e 2,00/1.000 NV, respectivamente) para 2015 (2,30/1.000 NV e 2,07/1.000 NV, respectivamente) e reduziram de 2015 para $2016(1,34 / 1.000 \mathrm{NV}$ e 2,06/1.000 NV, respectivamente); o Brasil reduziu seu valor de 2014 (2,05/1.000 NV) para 2015 (1,88/1.000 NV) e aumentou de 2015 para 2016 (2,21/1000 NV). Constatou-se que muito precisa ser desenvolvido na melhoria da qualidade da atenção primária, uma vez que a mortalidade pós neonatal está associada à deficiência na assistência ofertada às gestantes e na atenção aos recém-nascidos.

Palavras-chave: saúde da criança; mortalidade pós neonatal; atenção primária. 


\section{ABSTRACT}

Infant mortality, especially neonatal postpartum, is a sensitive indicator of the quality of life of a population, and it was therefore proposed to present the post-neonatal mortality coefficient of the city of Palmas, Tocantins state and Brazil, during the triennium 2014- 2016, in addition to explaining the main avoidable causes of these deaths. This is an exploratory descriptive study that analyzes public data provided by the Mortality Information System (SIM) and the Live Birth Information System (SINASC). The results presented as main factors related to postneonatal mortality, failures in diagnosis and treatment, promotion actions linked to primary care, attention to the newborn (NB), failures in the follow-up of gestation and delivery, and immunizations. There was a significant reduction in mortality in Palmas-TO, the opposite of the State and National scenarios. Mortality rates varied. Palmas and Tocantins increased their value in 2014 (1.55 / 1,000 live births (NV) and 2,00 / 1,000 NV respectively) to 2015 (2.30 / 1,000 NV and 2.07 / 1,000 NV, respectively) and reduced from 2015 to 2016 (1,34 / 1,000 NV and 2,06 / 1,000 NV, respectively); Brazil reduced its value from 2014 (2.05 / $1000 \mathrm{NV})$ to 2015 (1.88 / $1,000 \mathrm{NV})$ and increased from 2015 to 2016 (2.21 / $1000 \mathrm{NV})$. It was found that much is needed to be developed in improving the quality of primary care, since post-neonatal mortality is associated with deficiency in care offered to pregnant women and care for newborns.

Keywords: child health; postnatal mortality; primary attention.

\section{RESUMEN}

La mortalidad infantil, en especial la post neonatal, es un sensible indicador de la calidad de vida de una población, siendo así, se objetivó presentar el coeficiente de mortalidad post neonatal de la ciudad de Palmas, del estado de Tocantins y de Brasil, en el trienio 2014-2016, además de exponer las principales causas evitables de estas muertes. Se trata de un estudio descriptivo exploratorio que analiza datos públicos disponibilizados por el Sistema de Información sobre Mortalidad (SIM) y Sistema de Información sobre Nacidos Vivos (SINASC). Los resultados presentaron como principales factores vinculados a la mortalidad post neonatal la deficiencia en el diagnóstico y tratamiento, en acciones de promoción vinculadas a atención primaria, en la atención al recién nacido (RN), la gestación y el parto, e inmunizaciones. Se encontró una reducción significativa de la mortalidad en Palmas-TO, el opuesto de los panoramas Estadual y Nacional. Las tasas de mortalidad presentaron variaciones. Las Palmas y Tocantins aumentaron su valor de 2014 (1,55 / 1.000 nacidos vivos (NV) y 2,00 / 1.000 NV, respectivamente) para 2015 (2,30 / 1.000 NV y 2,07 / $1.000 \mathrm{NV}$, respectivamente) y redujeron de 2015 a 2016 (1,34 / 1.000 NV y 2,06 / 1.000 NV, respectivamente); Brasil redujo su valor de 2014 (2,05 / 1.000 NV) para 2015 $(1,88$ / $1.000 \mathrm{NV})$ y aumentó de 2015 a 2016 (2,21 / 1000 NV). Se constató que muy necesario ser desarrollado en la mejora de la calidad de la atención primaria, ya que la mortalidad post neonatal está asociada a la deficiencia en la asistencia ofrecida a las gestantes y en la atención a los recién nacidos.

Descriptores: salud del niño; mortalidad post neonatal; atención primaria.

\section{INTRODUÇÃO}

A mortalidade infantil é decorrente da associação de fatores sociais, culturais, biológicos e de falhas do sistema de saúde pública. Assim, componentes básicos, como saneamento, renda familiar, nível de instrução das gestantes e acesso aos serviços de saúde interferem diretamente nesse parâmetro, tornando a maioria das mortes infantis 
evitáveis e exteriorizando a qualidade da saúde pública no Brasil (ARAUJO, 2014).

A taxa de mortalidade infantil (TMI) é definida como o número de óbitos de crianças menores de um ano sobre o número de nascidos vivos (multiplicada por 1.000) e mostra o risco de um nascido vivo evoluir a óbito. Para o cálculo da TMI, considera-se a mortalidade neonatal (óbitos de 0 a 27 dias de vida) e a pós neonatal (28 dias a 1 ano de idade). A mortalidade neonatal é subdividida em neonatal precoce (óbitos de 0 a 6 dias completos de vida) e neonatal tardio (óbitos de 7 a 27 dias de vida) (FRANÇA \& LANSKY, 2008; ARAUJO, 2014).

O componente pós neonatal predominou no Brasil até o final da década de 80 (FRANÇA \& LANSKY, 2008), apresentando queda significativa a partir de 1990, de 24/1.000 nascidos vivos (NV), neste período, para 9,4/1.000 NV em 2000; decrescendo para 4,9/1.000 NV, em 2010. A partir de então, 70\% da mortalidade infantil está associada ao componente neonatal (GODOY \& GENOVESI, 2016).

A redução da taxa da mortalidade infantil tem sido atribuída a diversos fatores, como a queda da fecundidade, maior acesso da população ao saneamento e serviços de saúde, aumento da prevalência de aleitamento materno, às ações de imunização, antibioticoterapia e terapia de reidratação oral, entre outros (FRANÇA \& LANSKY, 2008).

Embora a TMI brasileira demonstre tendência decrescente com 78,5/1.000 NV em 1980, e 21,2/1.000 NV em 2005 (FRANÇA \& LANSKY, 2008), continua sendo um desafio atingir taxas ainda menores, principalmente quando considera-se as discrepâncias regionais do país (GODOY \& GENOVESI, 2016). A desigualdade socioeconômica influencia no acesso aos serviços de saúde, como prénatal, assistência ao parto e ao RN, com menor oferta desses serviços nos grupos com baixa renda (FRANÇA \& LANSKY, 2008).
A Classificação de Ortiz, amplamente utilizada no Brasil, prevê uma segregação para óbitos em menores de 1 ano de acordo com suas causas e a possibilidade de preveni-los. São divididas em causas não evitáveis, condições mal definidas e condições evitáveis. As causas não evitáveis são constituídas por óbitos que se relacionam a uma gama de doenças de alta letalidade, doenças do sistema nervoso central, vírus da influenza, desconforto respiratório do recémnascido e síndrome da morte súbita na infância. As condições mal definidas são aquelas vinculadas a outros transtornos originados no período perinatal. As condições evitáveis abrangem causas evitáveis por imunoprevenção, adequado controle na gravidez, adequada atenção ao parto, ações de prevenção, diagnóstico e tratamento precoces e causas reduzíveis por parcerias com outros setores (MALTA \& DUARTE, 2007).

O conceito de óbitos evitáveis ou sentinel events foi proposto em 1976, considerando que estas mortes poderiam ser reduzidas por medidas de saúde eficazes (HARTZ et al., 1996). Estes óbitos têm sido propostos como eventos sentinela, que indicam a necessidade de investigação e alertam para prováveis problemas relacionados ao acesso à serviços qualificados de saúde (ALVES et al., 2008).

Assim, o objetivo deste estudo foi apresentar o coeficiente de mortalidade pós neonatal de Palmas, Tocantins e Brasil, no triênio 2014-2016 e expor as principais causas evitáveis desses óbitos.

\section{MATERIAIS E MÉTODOS}

Trata-se de um estudo descritivo exploratório a partir de dados secundários obtidos no Sistema de Informações sobre Mortalidade (SIM) e Sistema de Informação sobre Nascidos Vivos (SINASC), voltado para grupos de indivíduos, visando a investigação dos fatores vinculados à mortalidade pós neonatal por causas evitáveis. 
Os dados foram coletados em novembro de 2017 no SIM, ferramenta desenvolvida pelo Ministério da Saúde, em 1975, com o objetivo de coletar dados sobre mortalidade no país tendo como fonte de informação a Declaração de Óbito (DO). Permite analisar e construir indicadores epidemiológicos que refletem a eficiência da gestão em saúde. Outra ferramenta utilizada para levantamento de dados foi o SINASC, implantado em 1990, para a determinação do número de crianças nascidas em determinado período (ROMERO \& CUNHA, 2016).

O critério de seleção para compor os dadosdeficiências na cobertura das informações de óbitos, pesquisados foram os óbitos pós neonatais evitáveisconfigurando $63 \%$ dos municípios com registro ocorridos no triênio 2014-2016, em Palmas, Tocantins einadequado, perdendo apenas para o Nordeste em Brasil. Estes óbitos representam um sensível indicador danúmeros de sub-registro, especialmente municípios qualidade de vida de uma população pois refletem resultadosmenores em questões demográficas. Deve-se ressaltar dos esforços empreendidos pelo setor de saúde, associadosa possibilidade de áreas com cobertura incompleta e ao desenvolvimento socioeconômico e a infraestruturaque, portanto, comprometam a validade do cálculo ambiental, fatores que, quando mal fundamentados, resultamestatístico de mortalidade, as políticas públicas de em desnutrição infantil e infecções a ela associadassaúde materno-infantil e ações dirigidas a este grupo (JÚNIOR et al., 2016).

O levantamento das taxas de mortalidade infantil pós neonatal (TMIPN) foi realizado utilizando-se a razão entre o número de óbitos pósneonatal sobre o número de NV, multiplicando-se por 1.000, para o cálculo das taxas anuais. Posteriormente, os valores encontrados foram expostos cronologicamente em um gráfico e em uma análise estatística descritiva, calculando-se a variação percentual do fator preponderante nas mortes infantis pós neonatais, a fim de comparar os valores encontrados em cada local estudado durante a análise temporal proposta.

O SIM divide os óbitos registrados em associados a causas evitáveis, mal definidas e outras causas. As causas evitáveis do componente pós neonatal, no SIM, são agrupadas em causas evitáveis reduzíveis por falhas em ações de imunização, na atenção à mulher na gestação e no parto, recém-
(CORBIN, 1989; GOULART, 2002; SZWARCWALD et al., 2002; MESSIAS et al., 2016).

Embora a obtenção dos dados secundários tenha cerne em sistemas de informação em saúde reconhecidos nacionalmente e internacionalmente, o registro é desigual nas regiões do Brasil. (SZWARCWALD et al., 2002; MESSIAS et al., 2016).

Em 2014, as taxas de mortalidade pós neonatal encontradas foram de 1,55/1.000 NV, 2,00/1.000 NV e 2,05/1.000 NV em Palmas, Tocantins e Brasil, respectivamente. Em 2015, foram de 2,30/1.000 NV, 2,07/1.000 NV e 1,88/1.000 NV em Palmas, Tocantins e Brasil, respectivamente. Em 2016, foram de 1,34/1.000 NV, 2,06/1.000 NV e 2,21/1.000 NV em Palmas, Tocantins e Brasil, respectivamente. Os dados foram apresentados na Figura 1. 
No ano de 2015, como observado na figura 1, houve um aumento da TMIPN em Palmas acima dos patamares das outras esferas, destoando da tendência geral, alavancado por mortes evitáveis por deficiência na atenção a mulher durante a gestação, que cresceu $300 \%$ em relação a 2014. Entretanto, nesse mesmo ano, houve uma redução significativa de $50 \%$ dos casos de mortes evitáveis por ações adequadas de promoção de saúde (atenção primária deficiente), que continuou em queda no ano seguinte, explicando assim a TMIPN de 2016, que atingiu um valor, em média, 37\% menor que as taxas nacionais e estaduais daquele ano, levando Palmas a um patamar significativamente menor que o das outras regiões analisadas.

Figura 1: Taxa de mortalidade pós neonatal em Palmas, Tocantins e Brasil, no triênio 2014-2016.

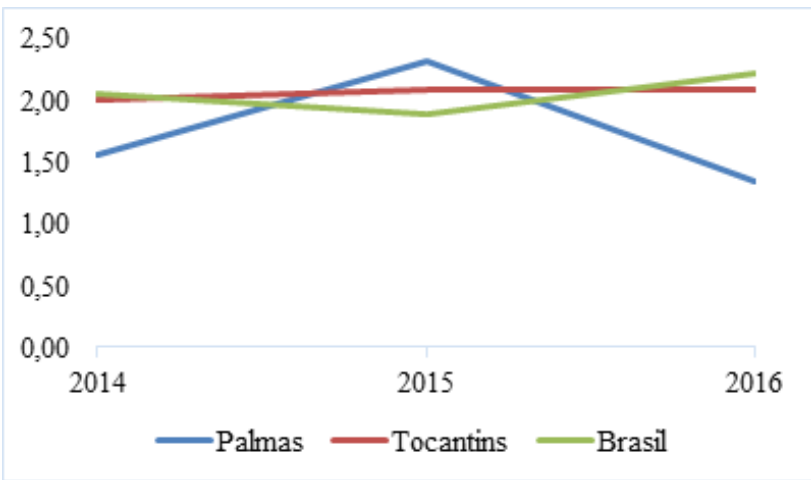

Fonte: SIM, SINASC, acessado em novembro de 2017.

O estudo Global Burden Disease Study 2015 (GBD, 2016) compara desempenho dos estados do país em relação a países com nível socioeconômico semelhante no setor associado ao percentual de declínio anual das taxas de mortalidade por todas as causas, a nível nacional, e afirma que os declínios mais acentuados se encontram nas regiões Norte e Nordeste do Brasil, enquanto os mais discretos localizam-se nas regiões Sul e Sudeste (BRASIL, 2010; SZWARCWALD et al., 2011), fator que pode estar associado ao avanço das coberturas de atenção ao pré-natal no país, que visa cada vez mais a qualificação do cuidado (VICTORA et al., 2011).
Neste estudo, comparadas as taxas de mortalidade infantil pós neonatal nacional, o município de Palmas apresentou valores inferiores em 2, dos 3 anos analisados. A queda das taxas de mortalidade infantil vincula-se às ações de promoção de saúde desenvolvidas a nível da atenção primária, em razão de que o componente pós neonatal da mortalidade infantil é reconhecidamente mais sensível às intervenções sanitárias e de saúde pública, como intervenção em quadros de diarreia, pneumonia, desnutrição, desidratação, anemia, entre outras (CALDEIRA et al., 2002).

No período analisado foram identificados, em 2014, 8 óbitos evitáveis em Palmas, 50 óbitos no estado do Tocantins; e 6.118, no Brasil. Em 2015, foram registrados 12 óbitos em Palmas, 52 no Tocantins e 5.641 no Brasil. Em 2016, ocorreram 6 óbitos evitáveis em Palmas, 45 no Tocantins e 5.474 no Brasil. No triênio, como mostrado na Figura 2, houve uma redução de $70 \%$ da taxa de mortalidade por atenção primária deficiente em Palmas e um crescimento em Brasil e Tocantins, apesar do aumento da TMIPN no ano de 2015 em Palmas.

Figura 2: Variação do item: taxa de mortalidade por Atenção Primária deficiente em Palmas, Tocantins e Brasil, no triênio 2014-2016.

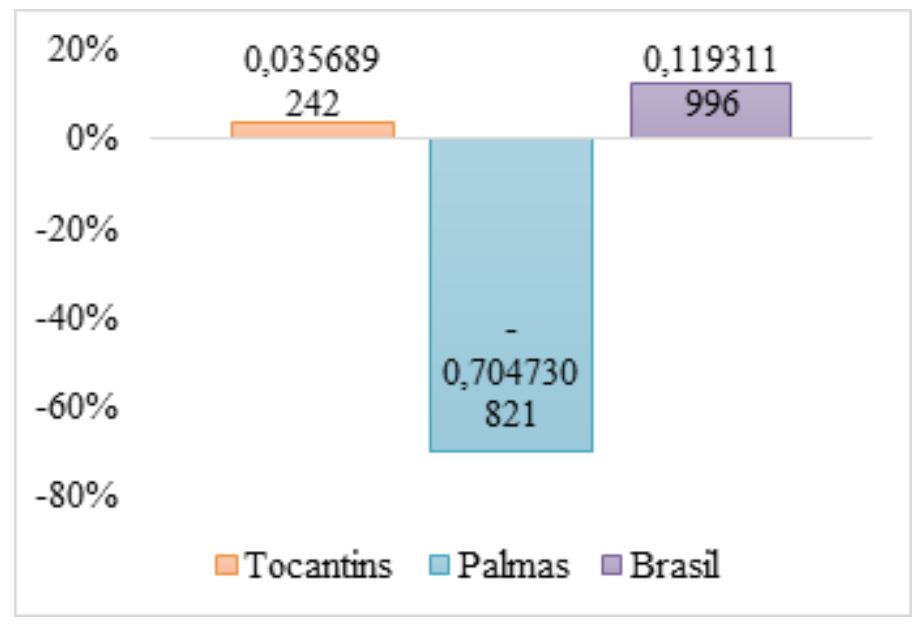

Fonte: SIM; SINASC, acessado em novembro de 2017.

Sabe-se que a mortalidade infantil tem forte vínculo com as condições socioeconômicas e ambientais, como a 
renda familiar, escolaridade dos pais, trabalho materno eencontrando dados na literatura que correlacionem a classe social, variáveis que, embora não causem doençasTMI do Tocantins e do município de Palmas com as diretamente, constituem determinantes distais que afetam ascondições socioeconômicas e ambientais, presume-se condições de vida, pois a inserção de classe determina aque a diferença na variação das taxas de mortalidade exposição da criança ao risco, bem como seu acesso àinfantil da capital do Tocantins frente ao Estado deveriqueza, estabelecendo os níveis de consumo e ase às diferentes condições socioeconômicas e disponibilidade de alimentação, moradia, saneamento,sanitárias e de acesso aos serviços de saúde.

assistência médica e escolaridade (FUCHS \& VICTORA, No estudo, as principais causas de óbitos 1997; CALDEIRA et al., 2002).

neonatais evitáveis encontradas nos anos de 2014 a

Um estudo de revisão realizado por Ferrari \&2016 em Palmas, Tocantins e Brasil estão expostos Bertolozzi (2012), no período de 2004 a 2009, no Brasil enas Tabelas 1, 2 e 3.

regiões (Sudeste, Centro-Oeste, Sul e Nordeste) sobre

mortalidade pós-neonatal no território brasileiro com oTabela 1. Causas de óbitos neonatais evitáveis, objetivo de identificar a relação da TMI neste componente ePalmas, Tocantins e Brasil, 2014.

sua relação com as condições socioeconômicas, identificoū Falha em: $\quad$ Palmas Tocantins Brasil

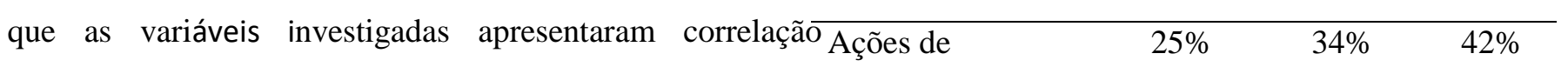
estaticamente significativa com a taxa de mortalidade diagnóstico e infantil, não sendo encontradas informações na região Norte tratamento do país.

Segundo dados do IBGE (2011), o Estado do Tocantins, criado em 05 de outubro de 1988, pertence à Região Norte do território nacional, é constituído por 139 municípios, e possui Palmas como capital. Oliveira e Piffer (2016) realizaram uma análise dos indicadores econômicos e sociais dos 139 municípios tocantinenses e como estes influenciaram o Índice de Desenvolvimento Regional (IDR) nos municípios do Estado do Tocantins, entre 2000-2010, sendo possível mensurar as desigualdades entre os municípios do Estado. Para isto, estimaram o IDR para os municípios, utilizando variáveis sociais e econômicas, tais como média de anos de estudo, leito hospitalar por habitante, percentuais de água encanada e de coleta de lixo, médicos por mil habitantes e despesas municipais com saneamento e saúde. Foi possível observar que o Estado do Tocantins apresentou profundos desequilíbrios sociais e econômicos em quase todos os municípios, com exceção de Palmas, Araguaína e Gurupi, estes últimos considerados municípios pólos. Nesta perspectiva, mesmo não

\begin{tabular}{|c|c|c|c|}
\hline $\begin{array}{l}\text { Ações de } \\
\text { promoção } \\
\text { vinculadas a } \\
\text { ações de atenção }\end{array}$ & $50 \%$ & $42 \%$ & $28 \%$ \\
\hline $\begin{array}{l}\text { Adequada atenção } \\
\text { ao RN }\end{array}$ & $12 \%$ & $8 \%$ & $13 \%$ \\
\hline $\begin{array}{l}\text { Adequada atenção } \\
\text { à gestação }\end{array}$ & $12 \%$ & $14 \%$ & $11 \%$ \\
\hline $\begin{array}{l}\text { Adequada atenção } \\
\text { ao parto }\end{array}$ & - & - & $4 \%$ \\
\hline $\begin{array}{l}\text { Ações de } \\
\text { imunizações }\end{array}$ & $0 \%$ & $2 \%$ & $2 \%$ \\
\hline
\end{tabular}

Fonte: SIM; SINASC, acessado em novembro de 2017.

Em seu estudo, Malta et al. apresentam como principais causas de morte pós neonatal no país, os óbitos por doenças transmissíveis, afecções maternas, neonatais e nutricionais, que podem ser considerados como preveníveis (MALTA et al., 2010). O presente estudo complementa o que o trabalho citado traz, uma vez que retrata como principais causas evitáveis falhas 
nos setores de ações de diagnóstico e tratamento adequados e ações de atenção para a população materno-infantil.

Tabela 2. Causas de óbitos neonatais evitáveis, Palmas, Tocantins e Brasil, 2015.

\begin{tabular}{lccc}
\hline Falha em: & Palmas & Tocantins & Brasil \\
\hline Ações de & $33 \%$ & $27 \%$ & $41 \%$ \\
diagnóstico e & & & \\
tratamento & & & \\
adequados & & $31 \%$ & $29 \%$ \\
\hline Ações de & & & \\
promoção & $17 \%$ & & \\
vinculadas a ações & & & \\
de atenção & & $14 \%$ \\
\hline Adequada atenção & $8 \%$ & & \\
ao RN & & & \\
\hline Adequada atenção & $33 \%$ & $19 \%$ & \\
à gestação & & & \\
\hline Adequada atenção & $8 \%$ & & \\
ao parto & & & \\
\hline Ações de & & & \\
imunizações & & & \\
\hline
\end{tabular}

Fonte: SIM; SINASC, acessado em novembro de 2017.

No estudo de Araújo et al., as principais causas redutíveis de óbitos no período pós neonatal são as falhas de diagnóstico e tratamento precoce e ações de promoção a saúde, associadas, em especial, a pneumonia, doenças infecciosas intestinais e deficiências nutricionais (ARAÚJO et al., 2010). Kumar et al., propõe para a redução desses óbitos a realização de ações de educação em saúde, em especial às mulheres em idade fértil, associada ao aumento do acesso pelas mesmas aos serviços de saúde e à qualidade no saneamento básico, fatores que estão diretamente envolvidos ao período pós neonatal, que reflete o início da interação da criança com a sociedade e o ambiente em que vive (KUMAR et al, 2010).

Logo, a aplicação destas propostas à realidade de Palmas, Tocantins e Brasil, considerando as principais causas de óbitos pós neonatais, poderiam se mostrar eficazes na redução dos valores das taxas de mortalidade.

Tabela 3. Causas de óbitos neonatais evitáveis, Palmas, Tocantins e Brasil, 2016.

\begin{tabular}{lccr}
\hline Falha em: & Palmas & Tocantins & Brasil \\
\hline Ações de & $50 \%$ & $31 \%$ & $40 \%$ \\
diagnóstico e & & & \\
tratamento \\
adequados \\
\hline $\begin{array}{l}\text { Ações de promoção } \\
\text { vinculadas a ações } \\
\text { de atenção }\end{array}$ & & & \\
\hline $\begin{array}{l}\text { Adequada atenção } \\
\text { ao RN }\end{array}$ & & & \\
\hline Adequada atenção à & $33 \%$ & $17 \%$ & $29 \%$ \\
gestação & & & $11 \%$ \\
\hline Adequada atenção & $0 \%$ & $4 \%$ & $4 \%$ \\
ao parto & & & \\
\hline Ações de \\
imunizações
\end{tabular}

Fonte: SIM; SINASC, acessado em novembro de 2017.

Quando se fala em deficiência em diagnóstico e tratamento de patologias, uma solução seria a qualificação dos profissionais da atenção primária e promoção da atenção na saúde materno-infantil. Em relação a deficiência da atenção na gestação, a realização do pré-natal voltado para identificação de mulheres que podem estar em maior risco para um desfecho adverso durante a gravidez, deve receber maior atenção por parte da equipe de saúde. Em relação à atenção ao $\mathrm{RN}$, as práticas de puericultura com controle do desenvolvimento infantil com registro dos progressos se faz de extrema importância 
para manejo da atenção em cada região (BRANDÃO et al., 2012).

No período de estudo, as variáveis que apresentaram relação com a mortalidade infantil pós neonatal em âmbito nacional, estadual e municipal foram aquelas vinculadas a cobertura e ações desenvolvidas na atenção primária de forma deficientes, sobretudo, falhas na promoção de saúde e prevenção de agravos voltados para o ciclo gravídicopuerperal e pós neonatal, além do conhecimento técnico-científico da equipe de saúde desatualizado, e emprego de condutas e tratamentos inadequados a este grupo. Contexto discutido por ARAÚJO et al. (ARAÚJO et al., 2010), que ressaltam a importância de investimentos em saúde nos momentos de gestação, parto e nascimento, uma vez que os óbitos pós neonatais possuem íntima relação com a qualidade da assistência pré-natal e neonatal.

Em conformidade com o estudo de Costa et al. (COSTA et al., 2003) e Duarte (DUARTE, 2007), programas e políticas de saúde pública, como os Programas de Atenção Integral à Saúde da Mulher (PAISM), Terapia de Reidratação Oral (TRO) e Programa Nacional de Imunização (PNI) corroboraram com o declínio da mortalidade infantil, desde a década de 80. Ademais, a mudança do perfil socioeconômico, demográfico, variação da fecundidade e índice de analfabetismo e as condições ambientais do brasileiro também contribuem para a queda da mortalidade (CALDEIRA et al., 2002; FERRARI \& BERTOLOZZI, 2012).

Como limitação do estudo, se reconhece o fato de utilizar dados secundários, os quais possuem desvantagens como, por exemplo, vieses de informações, pois o pesquisador não é responsável por manter, atualizar, fornecer e inserir os registros na base de dados. Tal afirmação é ilustrada pelo estudo de Albiero, L. G., \& Pereira, R. J.(2016) realizado em Palmas que verificou a existência de preenchimentos irregulares em declarações de óbito com informações ignoradas ou em branco. (ALBIERO, L. G., \& PEREIRA, R. J., 2016). Assim, é possível que os dados colhidos no presente estudo sofram interferência advinda de registros incompletos nos bancos de dados.

\section{CONCLUSÃO}

O conhecimento dos indicadores de morbimortalidade pós neonatal faz-se uma estratégia útil para detectar necessidades de saúde com o objetivo de subsidiar intervenções voltadas para a redução do risco de óbito, permitindo analisar e identificar os fatores associados às mortes, possibilitando ressaltar falhas ainda persistentes no sistema de saúde.

O estudo demonstrou uma redução na taxa de mortalidade infantil pós-neonatal por atenção primária deficiente em Palmas, diferentemente do Brasil e Tocantins. Os principais fatores associados à mortalidade foram falhas nas ações de diagnóstico e tratamento, atenção à gestante, ao recém-nascido e ao parto e imunizações, fatores que regem o binômio mãe-bebê e estão diretamente relacionados à gestão do cuidado da saúde materno-infantil pelos profissionais de saúde. Logo, fatores que auxiliariam a redução da mortalidade seriam o diagnóstico precoce, a qualidade da assistência, o acesso aos serviços primários de saúde e a disponibilidades destes serviços a nível nacional, associados a mecanismos de gestão integral para a saúde materno-infantil.

Constatou-se que muito precisa ser desenvolvido na melhoria da qualidade da atenção primária, uma vez que a mortalidade pós neonatal está associada à deficiência na assistência ofertada às gestantes e na atenção aos recém-nascidos. Soluções apresentadas foram investimentos nos profissionais de saúde, com foco na educação permanente, visando à constituição de uma equipe voltada para o componente pautado na integralidade do cuidado, melhorias na qualidade de consultas de pré-natal e 
puericultura, para um acompanhamento continuado que possibilite identificar patologias e intervir de forma precoce.

Todos os autores declararam não haver qualquer potencial conflito de interesses referente a este artigo.

\section{REFERÊNCIAS}

ALBIERO, L.G.; PEREIRA, R. J. Óbitos infantis em Palmas-TO e a sua relação com o uso de ácido fólico na gestação. Desafios, v. 3, n. 2, p: 123-131, 2016.

ALVES, A.C. et al. Principais causas de óbitos infantis pós-neonatais em Belo Horizonte, Minas Gerais, Brasil, 1996 a 2004. Rev. Bras. Saúde Matern. Infant., v. 8, n. 1, p: 27-33, 2008.

ARAÚJO, A.R.C. et al. Óbito em crianças menores de um ano: uma realidade de um serviço de urgência e emergência de São Luís - MA. Rev. Invest. Biom. Uniceuma, v. 2, p: 56-64, 2010.

ARAÚJO, J.P. et al. História da saúde da criança: conquistas, políticas e perspectivas. Rev. Bras. Enferm., v. 67, n. 6, p: 1000-1007, 2014.

\section{BRANDÃO, I.C.A.; GODEIRO, A.L.S.; MONTEIRO}

A.I. Assistência de enfermagem no pré-natal e evitabilidade e de óbitos neonatais. Rev. Enferm. UERJ., v. 20, p: 596-602, 2012.

BRASIL. Ministério da Saúde. Pacto pela redução da mortalidade infantil no Nordeste e Amazônia Legal: 2009-2010. Brasília: Ministério da Saúde, 2010.

CALDEIRA, A.P.; FRANÇA, E.; GOULART, E.A.M. Mortalidade infantil pós-neonatal evitável: o que revelam os óbitos em domicílio Rev. Bras. Saúde Matern. Infant., Recife, v. 2, n. 3, p: 263-274, 2002.
CAMPOS, T.P.; CARVALHO, M.S.; BARCELLOS, C.C.; Mortalidade infantil no Rio de Janeiro, Brasil: áreas de risco e trajetória dos pacientes até os serviços de saúde. Rev. Panam. Salud Pública, Rio de Janeiro, v. 8, n. 3, p. 164-171, 2000.

CORBIN, S.B. Improving Causeof-Death Statistics. Am J Public Health. v. 79, p: 563-564, 1989.

COSTA, M.C.N. et al. Infant mortality in Brazil during recent periods of economic crisis. Rev. Saúde Pública. v. 37, n. 6, p: 699-70, 2003.

DUARTE, C.M.R. Reflexos das políticas de saúde sobre as tendências da mortalidade infantil no Brasil: revisão da literatura sobre a última década. Cad. Saúde Pública [online]. v. 23, n.7, p: 1511-1528, 2007.

FERRARI, R.A.P.; BERTOLOZZI, M. R. Mortalidade pós neonatal no território brasileiro: uma revisão da literatura. Rev. Esc. Enferm, USP, v. 46, n. 5, p: 1207-1214, 2012.

FRANÇA, E.; LANSKY, S. Mortalidade Infantil Neonatal no Brasil: Situação, Tendências e Perspectivas. Abep [Internet], p:1-29, 2008.

FUNCHS, S.C.; VITORA, C.G. Técnicas de análise de dados para estudos de condições de vida e situação de saúde: análise hierarquizada aplicada a investigação de fatores de risco para agravos à saúde infantil. In Condições de vida e situação de saúde, p: 271-276. 1997.

G.B.D. Child Mortality Collaborators. Global, regional, national, and selected subnational levels of stillbirths, neonatal, infant, and under-5 mortality, 1980-2015: a systematic analysis for the Global 
HARTZ, Z.M.A. et al. Mortalidade infantil "evitável" em duas cidades do Nordeste do Brasil: Indicador de qualidade do sistema local de saúde. Rev. Saúde Pública, v. 30, n. 4, p: 310-318, 1996.

JÚNIOR, J.D. de P. et al.| Perfil da mortalidade neonatal no município de Ubá/MG, Brasil. Rev. Bras.

Pesq. Saúde, v. 18, n. 3, p: 24-31, 2016.

KUMAR, R. et al. Causes of neonatal and child mortality in India: a nationally representative mortality survey. Lancet, v. 376, n. 9755, p: 1853-60, 2010.

MALTA, D.C.; DUARTE, E.C.; Causas de mortes evitáveis por ações efetivas dos serviços de saúde: uma revisão da literatura. Cien. Saúde Colet. [Internet], v. 12, n. 3, p: 765-776, 2007.

MALTA, D.C. et al. Atualização da lista de causas evitáveis por intervenções do Sistema Único de Saúde. Epidemiol. Serv. Saúde, Brasília, v. 19, n. 2, p: 173-176, 2010.

MESSIAS, K.L.M. et al. Qualidade da informação dos óbitos por causas externas em Fortaleza, Ceará, Brasil. Cien. Saúde Colet. [Internet]. v. 21, n. 4, p:12551267, 2016.

OLIVEIRA, L. A. P.; MENDES, M. M. S. Mortalidade infantil no Brasil: uma avaliação de tendências recentes. In: Minayo MCS, organizadora. Os muitos Brasis: saúde e população na década de 80 . Hucitec Abrasco, São Paulo, 2a ed., p. 291-303, 1999.
OLIVEIRA, N. M.; PIFFER, M. Conjuntura do desenvolvimento regional dos municípios do estado do Tocantins. Desenvolvimento Regional em debate: DRd, v. 6, n. 3, p: 32-61, 2016.

ROMERO, D.E; da CUNHA, C.B.; Disponibilidade e qualidade da informação epidemiológica e demográfica no Sistema de Informações sobre Nascidos Vivos (SINASC) e no Sistema de Informação de Mortalidade (SIM) para menores de um ano. Anais. In: XV Encontro Nacional de Estudos Populacionais, ABEP, Caxambú-MG, p: 117, 2006.

SANTOS, E. P. et al. Mortalidade entre menores de um ano: análise dos casos após alta das maternidades. Rev. Bras. da Esc. Enferm. da USP.; v. 50, n. 3, p: 390-398, 2016.

SZWARCWALD, C.L. et al. Estimação da mortalidade infantil no Brasil: o que dizem as informações sobre óbitos e nascimentos do Ministério da Saúde? Cad. Saúde Pública [Internet]. v. 18, n. 6, p: 1725-1736, 2002.

SZWARCWALD, C.L. et al. Busca ativa de óbitos e nascimentos no Nordeste e na Amazônia Legal: Estimação das coberturas do SIM e do SINASC nos municípios brasileiros. In: Brasil. Ministério da Saúde. Saúde Brasil 2010: uma análise da situação de saúde e de evidências selecionadas de impacto de ações de vigilância em saúde. Brasília: Ministério da Saúde, p: 79-98, 2011.

VICTORA, C.G. et al. Maternal and child health in Brazil: progress and challenges. The Lancet. v. 377, n. $9780, \quad$ p: $1863-76, \quad 2011$. 